\title{
Methods of reconstructing complex multi-structural anatomical objects with RP techniques
}

\author{
T. KUDASIK* and S. MIECHOWICZ \\ Department of Mechanical Engineering, Faculty of Mechanical Engineering and Aeronautics, \\ Rzeszow University of Technology, 8 Powstańców Warszawy Ave., 35-959 Rzeszów
}

\begin{abstract}
This article presents reconstruction methods applied to a (geometrically and physically) complex structural object with the use of RP and RT techniques. The methods are innovative due to their hybrid - multi-model and multi-material - approach to reconstruction, as well as the application of multiple technologies. An experimental analysis was conducted to verify the feasibility of rapid prototyping (RP) techniques in the reconstruction of complex internal structures using materials of diverse properties. Some RP techniques offer the possibility of discriminating between diverse objects through the use of different colours. Such models are well-suited for diagnostic purposes, for better visualisation of complex clinical problems, pathological alterations, etc. Nevertheless, they fail to fully reflect physical and mechanical properties of objects, which renders them useful in experimental analysis only to a limited extent. Their basic drawback is that they merely reflect geometrical features of the examined object. The methods discussed in the present article enable modelling multi-object structures in a single process based on the PolyJet Matrix technology and materials of different physical properties by means of a hybrid method. The article also describes the process of modelling complex anatomical structures of soft tissues and bones using models of the maxilla and the mandible as examples. The study is based on data acquired through standard computed tomography (CT). In addition, the article addresses selected aspects of CT acquisition, generation of numerical models composed of several anatomical structures (objects) and fabricating physical multi-object models.
\end{abstract}

Key words: medical modelling, rapid prototyping, computed tomography (CT), experimental tests.

\section{Introduction}

The increasingly expanding scope of modern medical imaging technologies (computed tomography, magnetic resonance tomography, ultrasonic imaging) has opened new possibilities for high-quality visualisation of clinical cases. Conventional X-ray diagnostics is rather ineffective when imaging objects of complex structure, especially with a large number of anatomical details, i.e. hard and soft tissues of the craniofacial. Conventional methods offer low level of precision in visualising bone lesions in periodontal conditions, functional disorders of temporomandibular joints or pathological alterations in soft tissues of the face and the oral cavity. The reason stems from the very nature of the tests, which consist in projecting three-dimensional anatomical structures on the plane of an X-ray film. Anatomical interpretation of data in the form of a flat projection of the image is difficult and requires considerable experience. The most significant shortcomings of X-ray imaging (2D) include the superimposition of tissue structures, distortions due to single projection and differences in the density of adjacent regions occupied by various tissues (Fig. 1). For this reason, analyses of more complex medical issues make increasing use of digital diagnostic imaging techniques such as computed tomography (CT) and magnetic resonance tomography (MRI) [1, 2].

Such techniques make it possible to obtain cross-sections of tissue structures in any plane (MPR: multi-planar reconstruction). 3D models of the test object (or multiple test objects) can be generated from a set of raster data (DICOM data). Vir-

*e-mail: tkudasik@prz.edu.pl tual models (3D numerical models) can be developed with the use of CT or MRI data. They can be useful in designing and fabricating physical models of real tissues by means of rapid prototyping techniques $[3,4]$. 3D models generated by the authors of the present study are shown in Fig. 2; they are based on anatomical structures presented in Fig.1.

RP methods enable us to create physical models for medical purposes [5] such as:

- maxillofacial and dental surgeries,

- orthopaedic and spinal surgeries,

- oncology and reconstruction surgeries,

- customised joint replacement prosthesis,

- patient-specific instrumentation (orthoses),

- implant design, testing and validation.

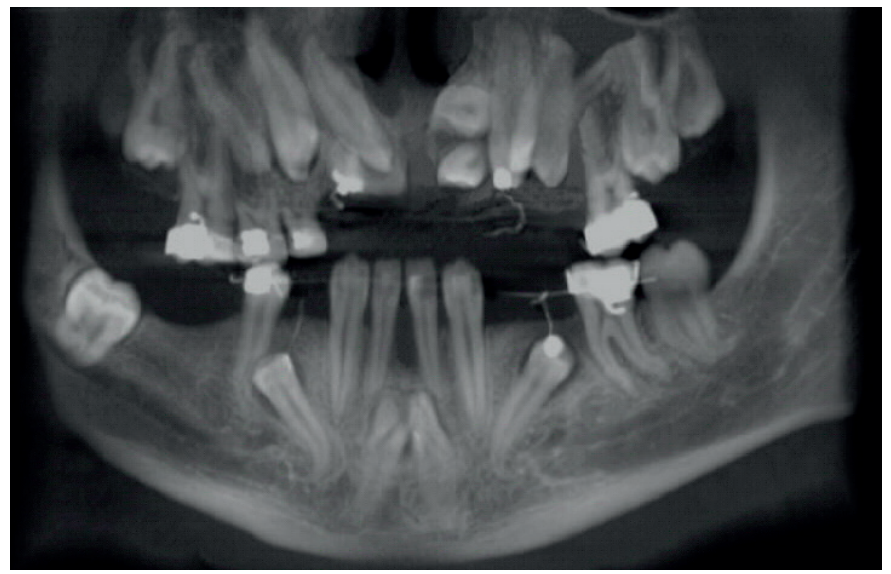

Fig. 1. A 2D example of grey-scale X-ray imaging of a section of the maxilla and the mandible (with barely visible complex bone structures) 

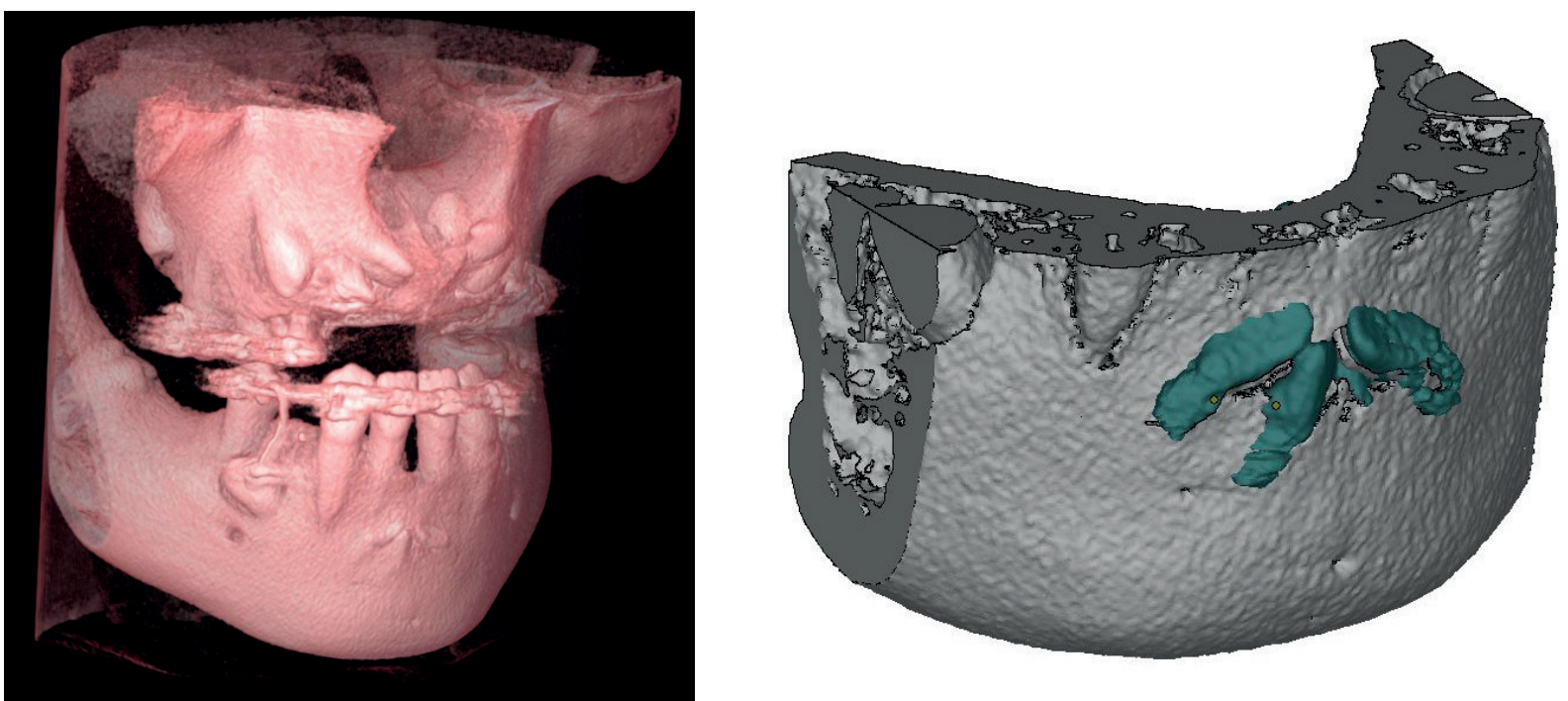

Fig. 2. Virtual 3D multi-object models of the maxilla and mandible sections (complex internal bone structures partly visible)

The most common applications of RP models are: preoperative planning for surgery $[6,7,8]$ and the preparation of surgical guides, templates, soft appliances $[9,10]$. The more complicated models are used for custom prosthetics, implant designing $[11,12,13]$ and scaffolds generation $[14,15]$. Medical RP models can also be utilised in research and experimental tests $[16,17,18]$. Some RP techniques offer the possibility of discriminating between diverse objects through the use of different colours of the printed material. Such models are well-suited for diagnostic purposes, for better visualisation of complex clinical problems, pathological alterations, etc. The PolyJet Matrix technology [19] offers the possibility of fabricating multi-part structures composed of various materials within the same object. This fabrication method can be applied in constructing hybrid models of bones and soft tissues in a single technological process. In the case of medical objects, data may be acquired via computed tomography, magnetic resonance imaging and angiography. Commonly used spiral CT can serve as a source of data for preparing multi-object anatomical models $[20,21]$.

The research aim of the present work was to develop a method for the reconstruction of a (geometrically and physically) complex structural object by means of RP and RT techniques. The methods are innovative in their hybrid (multi-model and multi-material) approach to reconstruction as well as the application of multiple technologies. The method developed allows us to apply materials of properties similar to those of real anatomical structures. Potential application of reverse engineering and rapid prototyping (RP) technologies in generating multi-structural medical models for experimental tests is shown below.

\section{Materials and methods}

2.1. A method of reconstructing complex multi-object tissue structures. In order to reconstruct geometrical features of soft tissues and bones, CT data were acquired. A segmentation method for data from complex medical objects was designed. Physical models were created using the RP technique (PolyJet Matrix) on the basis of high-resolution tomographic data from a patient.

CT data were obtained by scanning a 38-year old patient. The area of interest included parts of the maxilla and the mandible with extensive dental defects. The scans were completed on a Siemens Somatom Sensation 16 CT scanner. Selected dedicated measurement protocols were applied. The scan setup was as follows:

- collimation $0.6 \mathrm{~mm}$,

- slice thickness $0.6 \mathrm{~mm}$,

- table increment $0.5 \mathrm{~mm}$,

- reconstruction kernel U80u,

- matrix resolution 512 x 512 pixels,

- test area containing selected sections of the maxilla and the mandible.

Only a section of the region was selected so as to obtain the highest possible spatial resolution with acceptable noise level for reconstructing soft tissues. As air is the best contrasting medium in CT imaging, the method applied involved stabilizing patient in a way that would enable uninhibited access of air to all cavities in the surface of both soft tissues (mucous membrane) and teeth. The advantage of the proposed method is limiting the effect of artificial contrast media, the use of which is not necessary in the experiment described. The drawback of the approach involves difficulties in appropriately positioning the patient on the CT scanning table. The positioning should be very precise in order to achieve a stable and accurate position relative to the $\mathrm{CT}$ gantry. As the positioning is very complicated, the results of the acquisition rely partly on the patient's cooperation. Artificial elements, implants, fixing screws and other objects may cause visual artefacts. Image distortions may affect the quality of 3D model reconstruction.

CT images saved in the DICOM file format were imported in the 3D Doctor application (Able software) [22]. Three-di- 

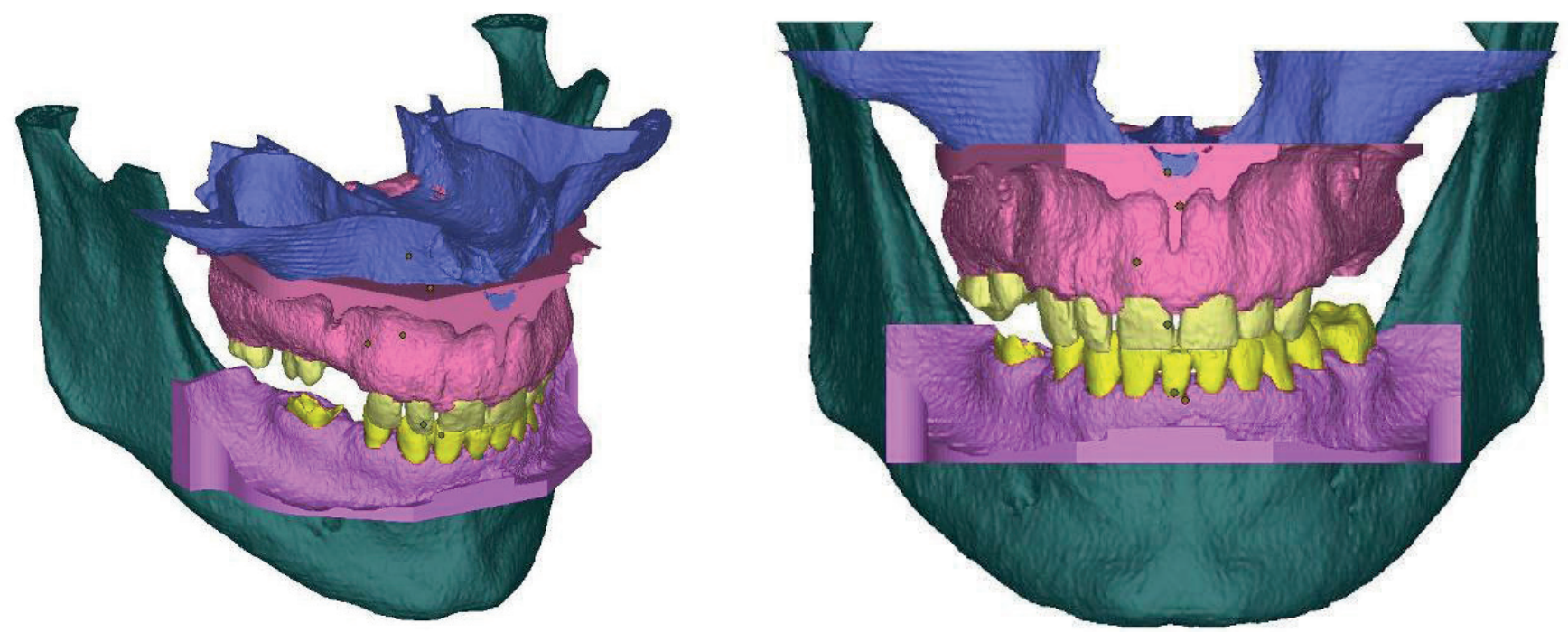

Fig. 3. Multi-object anatomical models of the maxilla and the mandible

mensional models of the maxilla and the mandible were generated (Fig. 3) on the basis of the segmentation of three different regions: bones, teeth and soft tissues. Virtual (3D) models were utilised to generate Objet Connex 500 [23] machine codes for separate tissue models. Different tissue areas were built in a single production process from silicone and resin materials. Soft tissue was reconstructed from TangoGray, bone tissue from FullCure 720, and teeth from NeroWhite (Fig. 4) materials.

Conventional methods applied in reconstructing the maxilla and the mandible entail a time-consuming process performed in several steps: 1-2 data acquisition (s) and fabrication in 2 or 3 stages. The proposed approach is less complicated due to its single-stage character. No additional impressions or further processing are necessary. The model is constructed using a sin- gle machine in a single production process. The limitations of the method include strict requirements in terms of the accurate placement and positioning of the patient on the CT table.

2.2. A method of reconstructing medical objects with internal structure from materials of diverse properties. The creation of a medical model is a complex process. Its broad scope of application requires selecting suitable production technologies in order to achieve the desired functionality of the model. The hybrid method is set out to fabricate a medical model of structure, properties and features which cannot be obtained by means of the direct method in a single production process, e.g. with RP techniques. The range of application includes reconstructing objects from materials of different baseline properties, modelling complex geometrical features, e.g. hollow internal
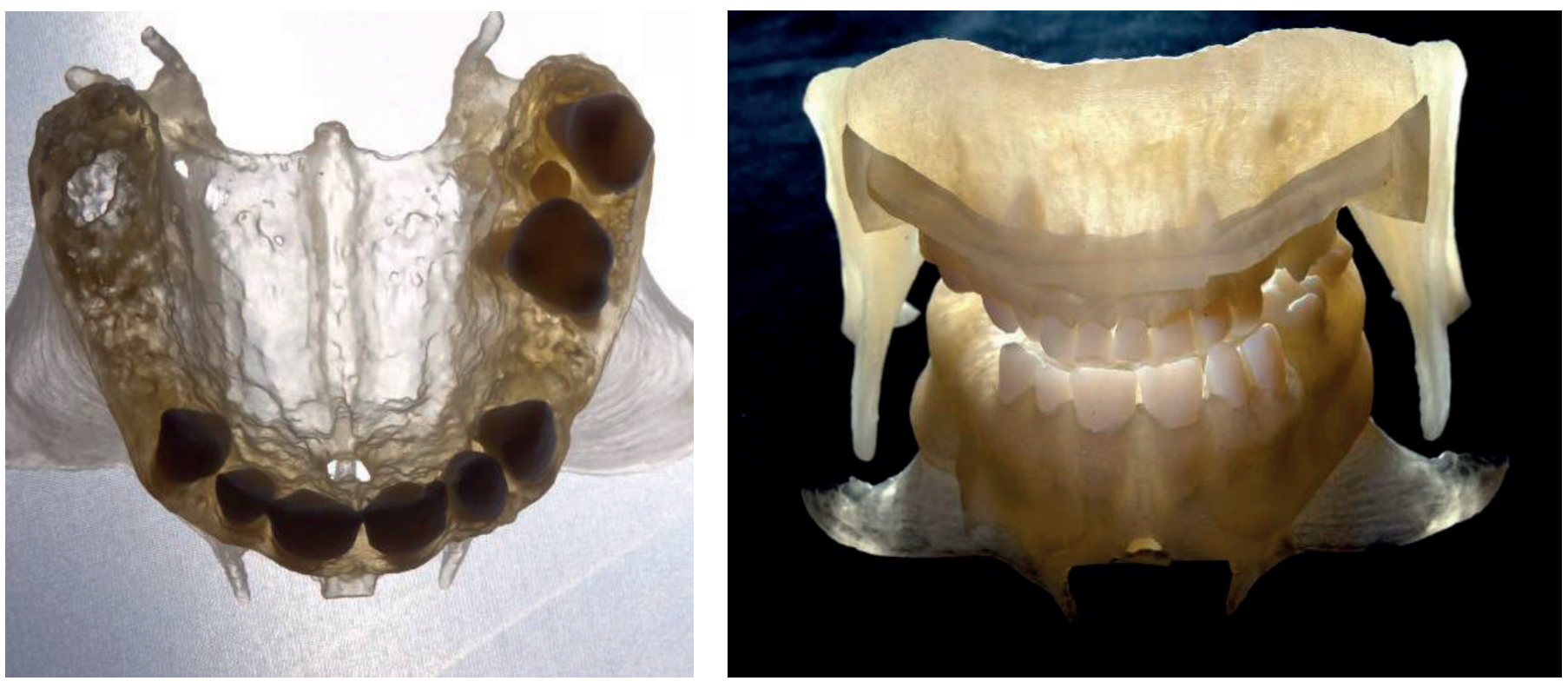

Fig. 4. RP model reconstruction by means of the PolyJet Matrix technology (Objet Connex500 printer) 

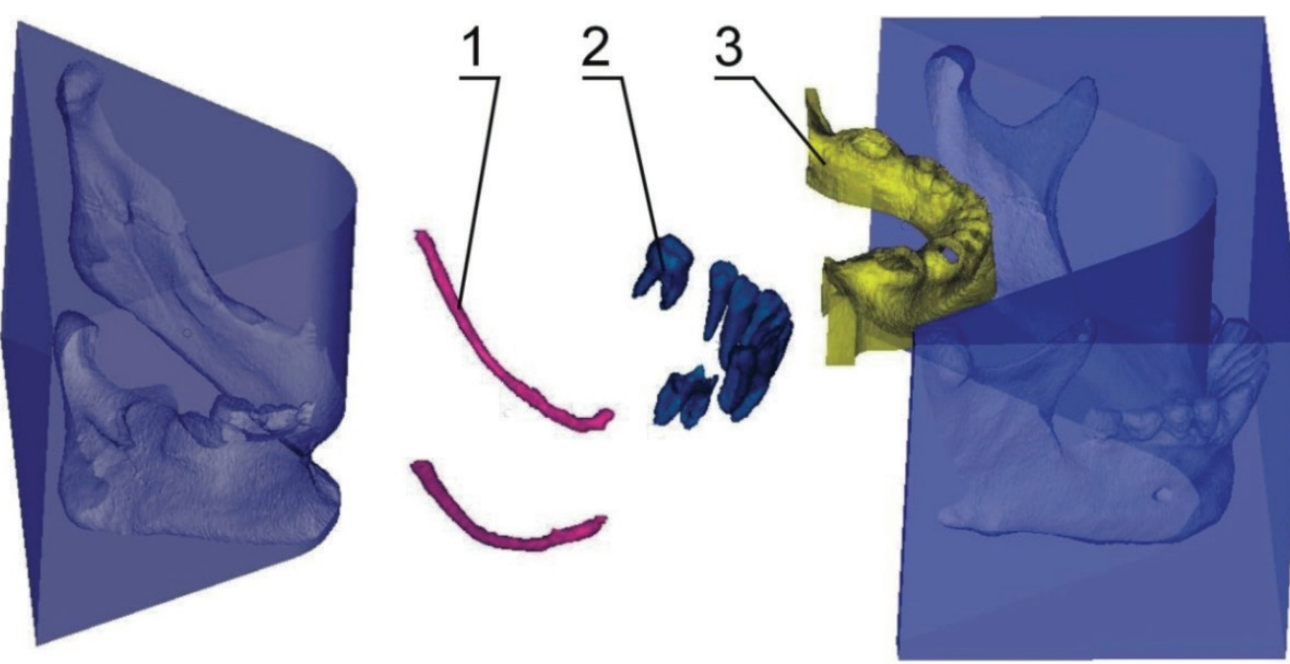

Fig. 5. A visualisation of a mandible silicone mould with components fixed in the silicone mould prior to the introduction of the bonding material: 1) a wax model of the nerve canal, 2) tooth models, 3) a silicone model of soft tissue

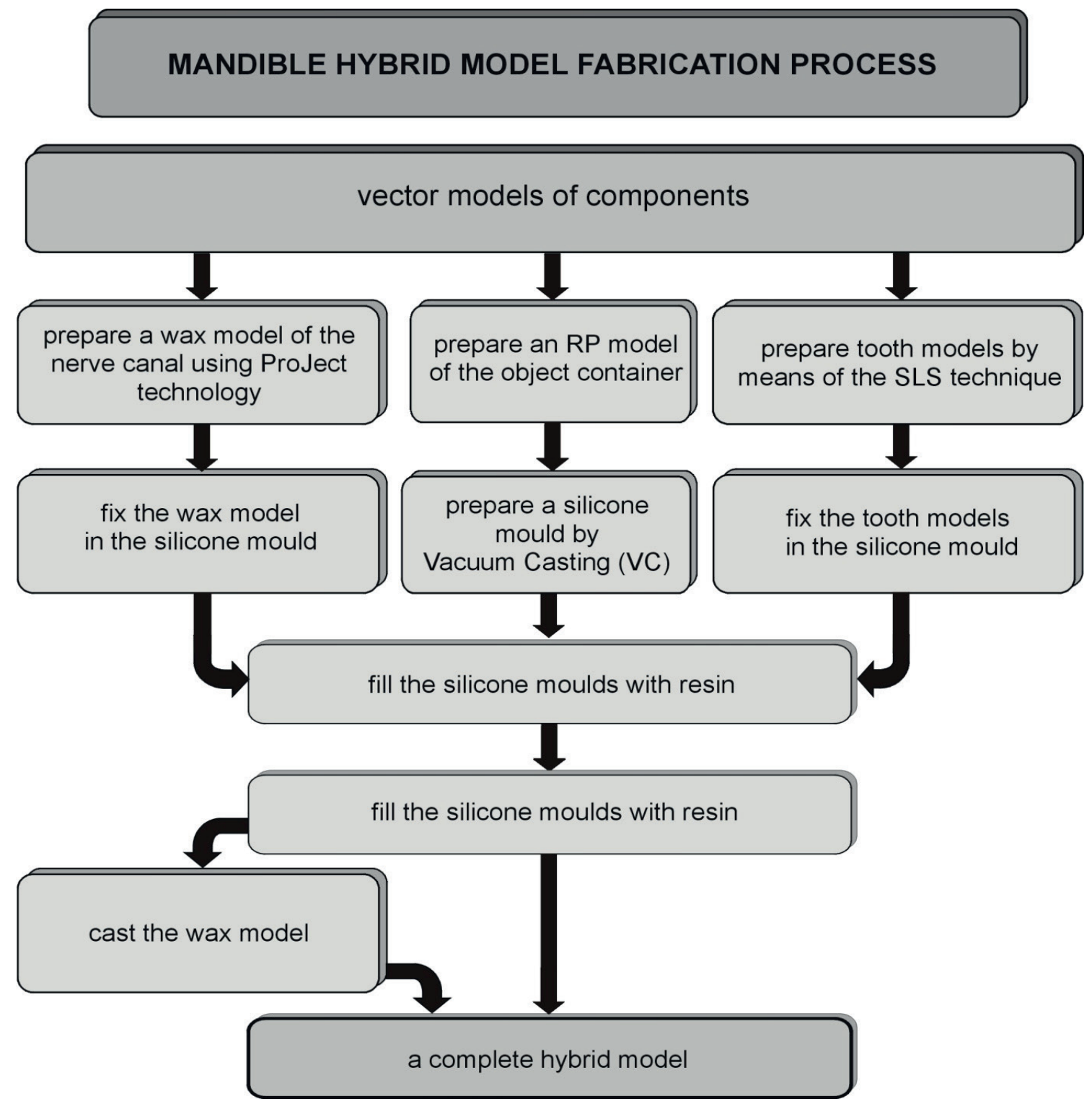

Fig. 6. Stages of model fabrication with the hybrid method 
structures, multi-structure objects etc. The approach assumes the possibility of making a model composed of several objects of different required material properties and internal structure. The approach may make use of objects constructed by means of RP and RT techniques. Figure 5 contains a visualisation of sample objects of the hybrid model.

The hybrid method enables us to fabricate an object of properties different from those obtainable with RP techniques. Another significant characteristic of the hybrid method is the possibility of re-using certain objects, e.g. moulds. This is of special importance in experimental studies in view of the reduction of the costs of fabricating models for experiments. Figure 6 is a flowchart detailing the process of fabricating a hybrid model of the mandible. Individual steps of the process are selected depending on the requirements to be met by the medical model.

Modelling the mandibular nerve canal was described below as a sample application of the hybrid method for reconstructing an object from a material of different initial properties and reconstructing complex internal geometrical features.

2.3. Reconstructing complex geometrical structures from materials of different properties. In experimental studies in which medical models are used, it is crucial to accurately recreate anatomical structures. This concerns not only their external shape, but also exactly copying the internal structure of tissues. For photoelasticity studies using reflected light, where stress distribution on the surface of the model is analysed, precise reconstruction of internal structure does not significantly affect measurement accuracy [24]. In photoelasticity tests using transmitted light, in particular with stress-freezing techniques [25], physical reconstruction of the model's internal structure is necessary. As the adopted approach makes it possible to analyse stress distribution within the model, the accuracy of copying the internal structure is very important. Methods which enable fabricating spatial models with internal structure on the basis of a virtual (3D) model include rapid prototyping (RP) techniques, especially SLA [16, 17] and JS - PolyJet [18, 19]. Experiments reveal that materials used in such methods have inferior photoelastic properties than typical epoxy resins used in photoelasticity studies, i.e. Epidian 51. Although the photoelastic effect is clearly visible, in some cases it may prove insufficient [26]. Epidian 51 is applied in moulding methods in which there is no possibility of recreating such intricate internal structure as that of e.g. bone tissue. This problem may be partly solved by wax casting. An accurate wax model including internal structure may be built by means of the JS - ProJet method. A sample section of the cranial suture in 4:1 scale fabricated on the CPX ProJet 3000 machine is shown in Fig. 7. A model of the suture fabricated with this approach reconstructs the internal structure of bones together with a fragment of the joint.

The wax model provides a basis for building a model from a biocompatible material or a material with properties suitable for experimental studies.

The wax model can be used to make silicone moulds by vacuum casting (VC). When the wax model has been cast, a structure reflecting both external shape and hollow cavi-
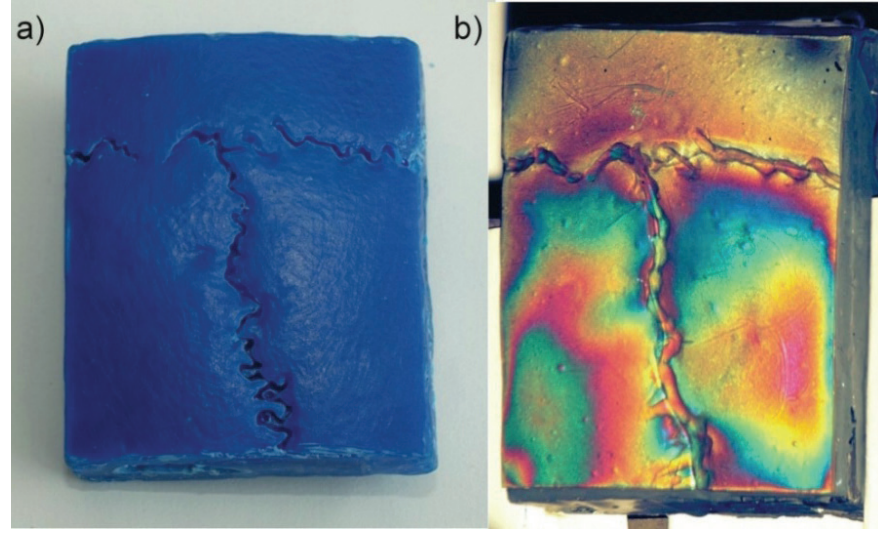

Fig. 7. A sample section of a cranial suture: a) RP model scale fabricated on the CPX ProJet 3000 machine, b) experimental model (photoelastic tests)

ties inside the bone is created inside the mould. A mould prepared in this manner can then be filled with e.g. epoxy resin in order to obtain the target model. As part of the moulding compound (silicone) forming the internal structure remains inside the model, it is necessary to cut off fragments of the internal structure when separating the model from the mould. Therefore, the method does not allow us to re-use the silicone mould. The wax casting approach makes it possible to obtain a model of the suture which is geometrically similar to the input model, and, more importantly, has better photoelastic properties. Figure 8 contains an image of a finished model of a section of the cranial suture fabricated by moulding from the Epidian 51 resin.

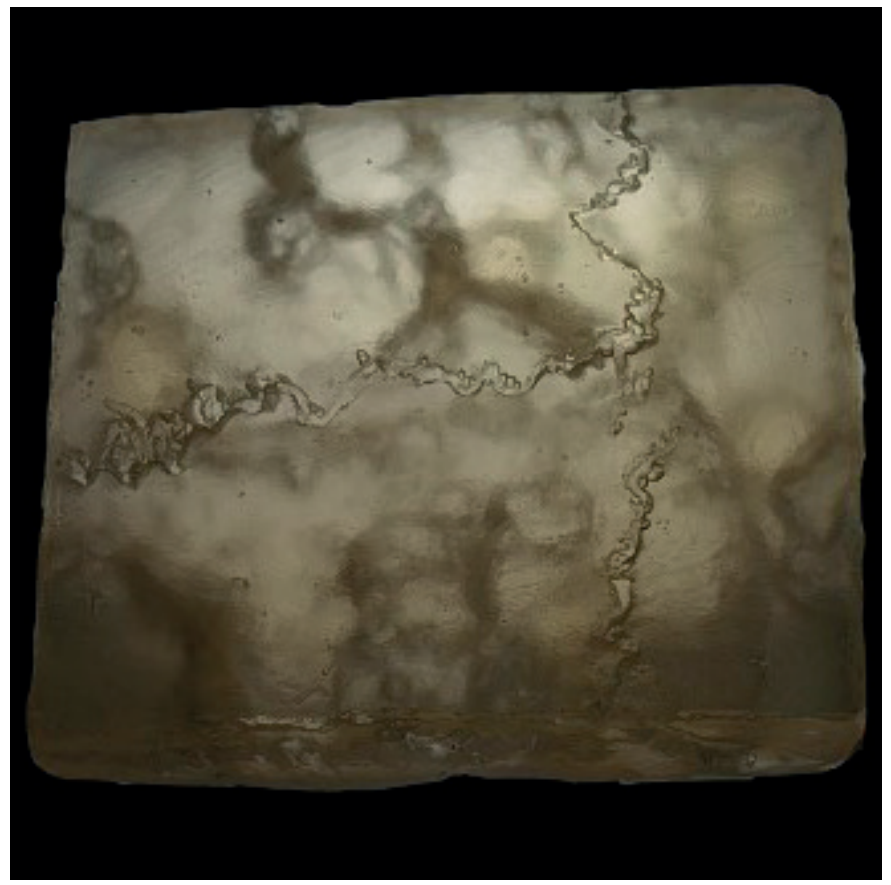

Fig. 8. A model of a section of the cranial suture fabricated by moulding from the Epidian 51 resin 

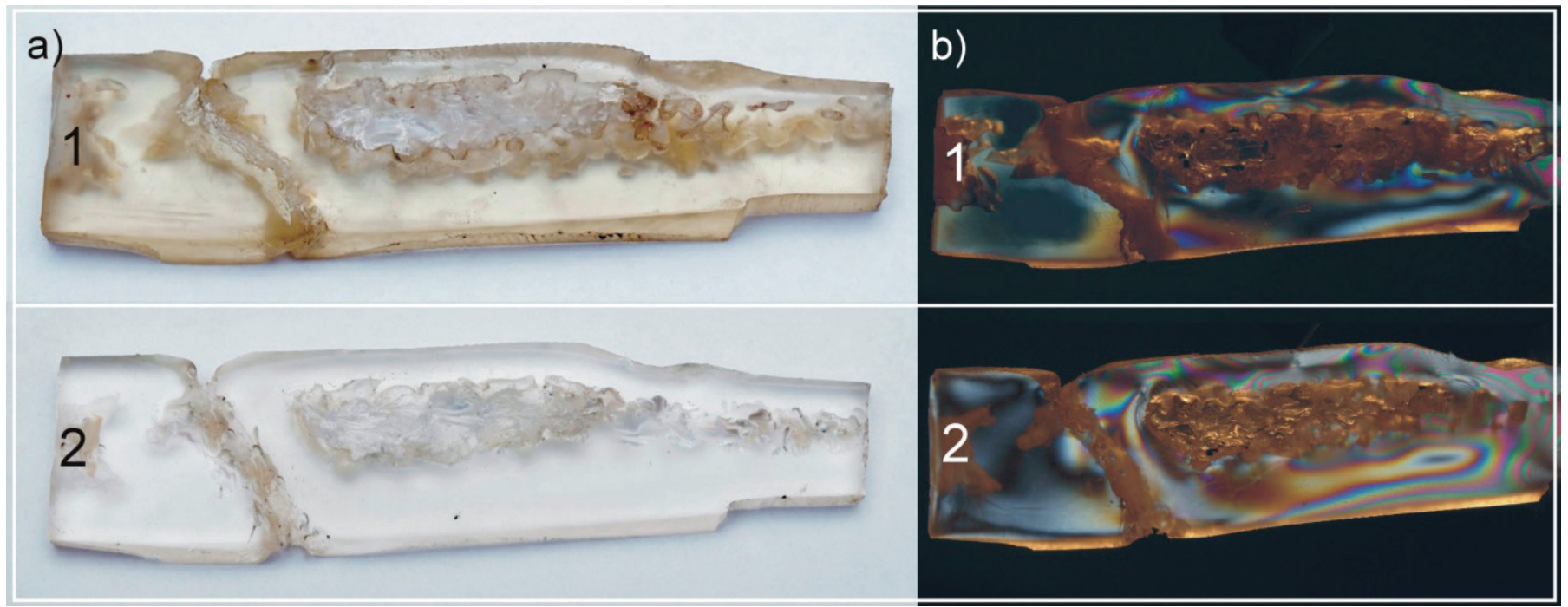

Fig. 9. Sample slices from a 3D model of a cranial suture a) RP (1. PolyJet technique) and VC (2. Epidian51) models, b) experimental tests (stress pattern)

The present model may be used in photoelastic test based on the reflected light approach. In the case of the transmitted light method, it is necessary to make section samples from the selected area. Figure 9 shows an example of sections of the bone structure in the form of thin-walled slices obtained by bisecting the cranial suture model fabricated with the PolyJet method and casting method. One can notice a high degree of similarity in reconstructing both external shape and internal structure of the item [21].

\subsection{Reconstructing hollow internal structure (the case of} the mandibular nerve canal). An important advantage of the hybrid method is the capability to fabricate anatomical models with internal structure from materials which are unobtainable by RP techniques.

Steps involved in the production of a model of the mandible containing an internal nerve canal with the application of the hybrid method are shown below. The 3D model of the mandible and the nerve canal was obtained from the acquisition of CT data. The model of the nerve canal (Fig. 10) reconstructs hollow space inside the mandible bones. The remaining components required to build the model are shown in Fig. 5.

The first stage of the process is constructing a silicone mould for the coat containing components of the hybrid model of the reconstructed object (object container). In order to obtain a silicone mould, an RP model was used, recreating external surfaces of the object - in this case, bones of the mandible (Fig. 11). The RP model does not need to map the internal structures or be transparent. However, for better visualisation of the process we used a model built with the SLA (Stereolitography) technique.

The silicone mould (Fig. 12) provides a temporary fixation for the components of the hybrid model. Internal surfaces of the model are obtainable by means of the ProJet wax modelling method. Sections of internal geometrical structures (nerve canals, sinuses, etc.) formed in wax are placed in the silicone mould.

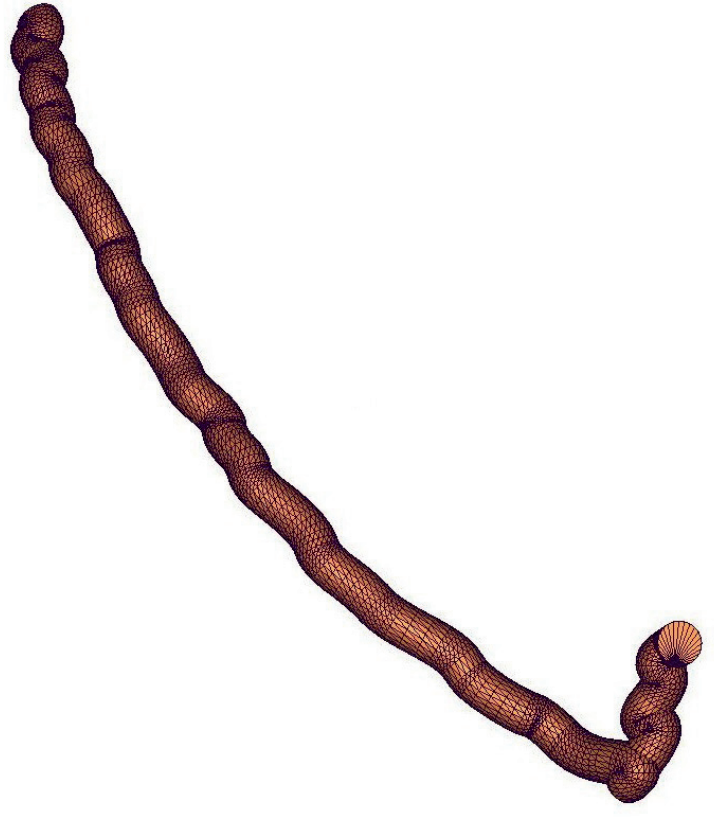

Fig. 10. A 3D model of the mandibular nerve canal

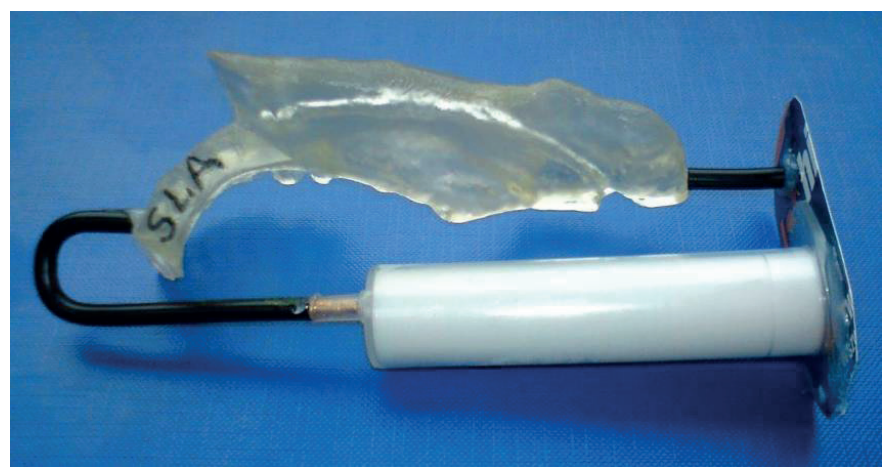

Fig. 11. An RP model of the mandible together with the mould set-up, prepared for filling with silicone 


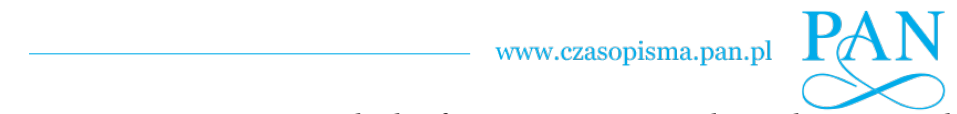

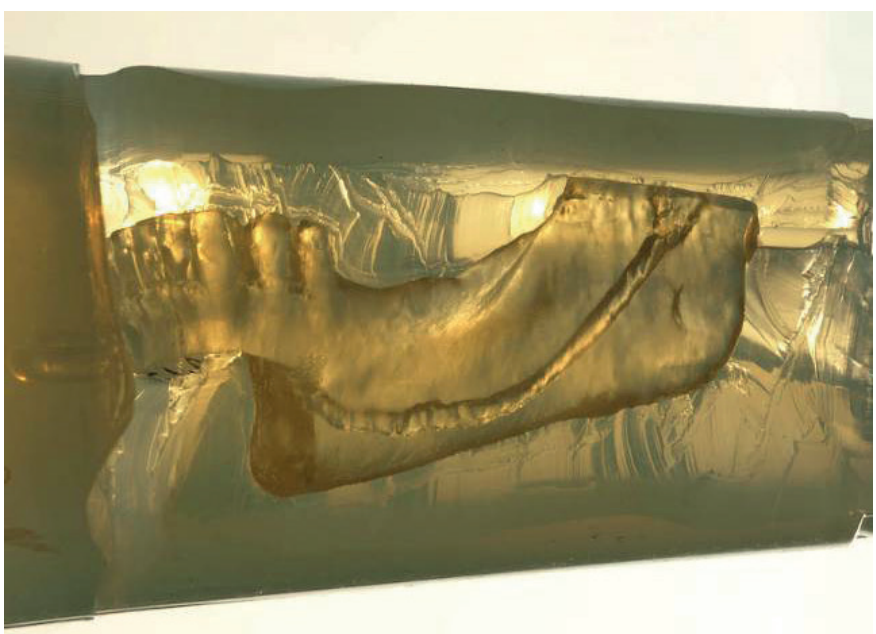

Fig. 12. An RP model of the mandible in a silicone mould

Another key step following the fabrication of internal structures is the correct placement of the supports in the mould. The supports should enable the inclusion of process allowances and casting the wax from the finished model (Fig. 13) later on.

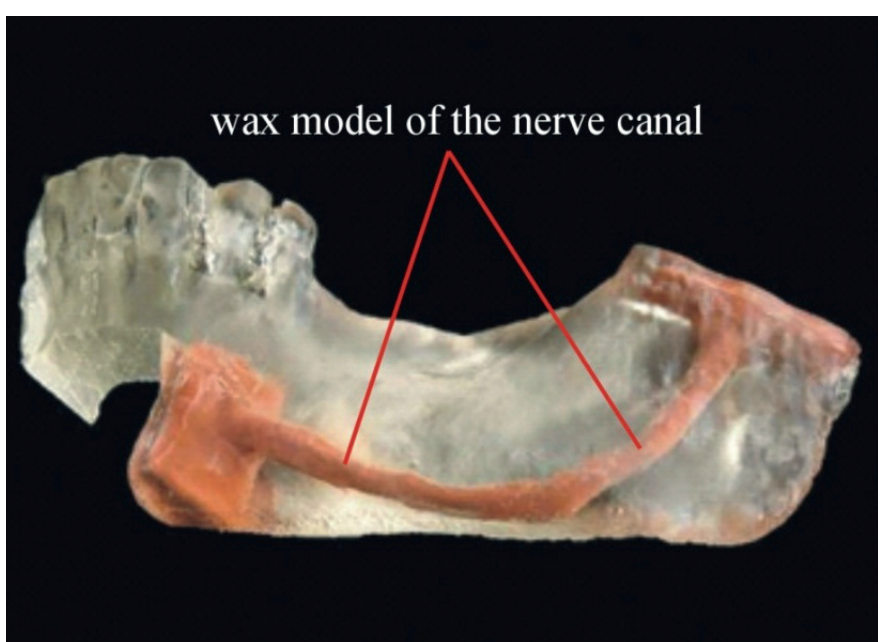

Fig. 13. A model of a section of the mandible fabricated by vacuum casting the Epidian 51 resin

A model of a section of the mandible with a cast mandibular nerve canal is presented in Fig. 14. The Epidian 51 resin has good photoelastic properties. Reconstructing a hollow nerve canal in the experimental model enables us to allow for the effect of internal structures in experimental studies on anatomical structures.

\section{Summary}

As in many other areas of scientific research, the information obtained in different experimental conditions can lead to uncertainty in the correct clinical interpretation and approach to be

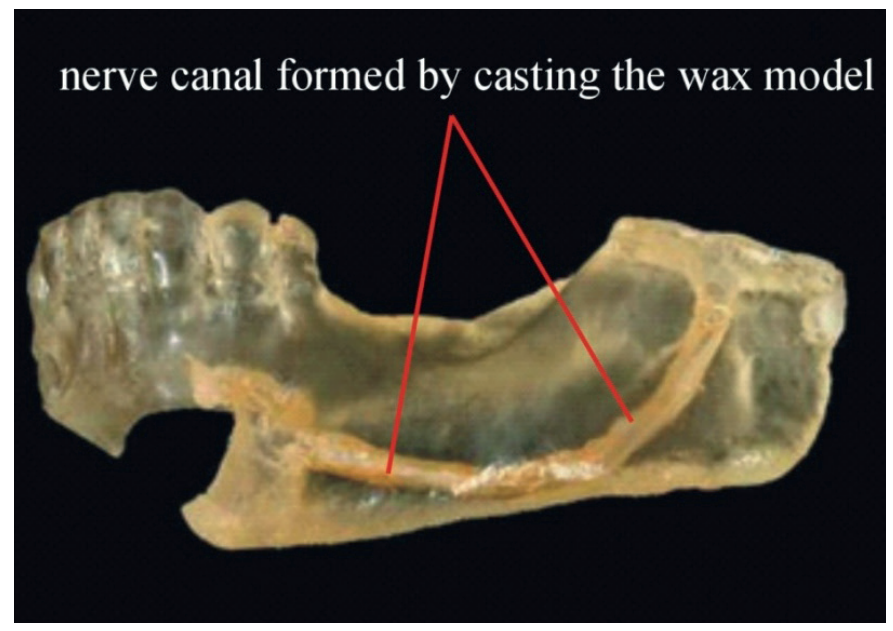

Fig. 14. A complete model of a section of the mandible fabricated by vacuum casting Epidian 51

taken. The results of numerical analyses on virtual 3D models of medical objects may be questionable, as the implementation of in vivo data obtained from humans can be challenging. For the analysis of complex medical issues, beside numerical methods, one can simultaneously make use of experimental tests to verify the obtained results. In the case of the experimental test, it is necessary to make physical models and to verify them by carrying out relevant tests. A physical model of the examined object can be fabricated with Rapid Prototyping technology from a 3D-CAD virtual model. Physical Rapid Prototyping model enables us to effectively identify the medical problem and clearly reconstruct pathological alterations $[1,7]$.

Multi-object and multi-material hybrid models may facilitate planning surgical procedures and allow patients to better understand the nature of the condition and the adopted course of treatment $[6,7,8]$. Based on CT and MRI data of real anatomical structures, it is possible to manufacture physical models which reconstruct complex inner structures very precisely [2, $20,27]$. It allows us to avoid simplified assumptions in experimental analysis. When making hybrid models, the selection of a suitable reconstruction protocol from the CT image is crucial for obtaining the desired results.

Experimental research may use real objects or their models. The photoelastic method can be used to verify the numerical analysis of the stress distribution in 3D models.

Material selection criteria are dependent on the type of experimental tests. In the case of photoelasticity studies (e.g. transmitted light studies) it is necessary to use transparent materials which guarantee the occurrence of forced birefringence, resulting in the appearance of isochromes indicating areas of stress. The materials used in experimental analysis were verified in terms of their photoelastic properties. The experiments allowed us to conclude that casting resin Epidian 51 offers the best photoelastic properties [26]. The necessity of using casting resin implied the preparation of the present hybrid method. In the experiments it was reported that the inclusion of internal structures made of various materials has a significant effect on the stress pattern (Fig. 9). 
The developed hybrid approach produces anatomical models with internal structure from materials of suitable photoelastic properties (transparency, birefringence) required in the transmitted-light photoelastic method, which are absent from materials used in RP techniques.

The methodology of fabricating multi-object models by combining RP and vacuum casting (VC) techniques was successfully verified using an experimental model of the mandible.

Due to the nature of the $\mathrm{VC}$ technology, it is essential to carefully select the physical properties of component materials (e.g. melting point, or softening point in the case of thermoplastic materials). Casting a bone model from the Epidian 51 resin triggers a chemical reaction during the polymerisation process, which may damage the model's components.

In most cases, the VC technique offers the possibility of re-using silicon moulds. However, this depends heavily on the correct planning of the dividing lines of the moulds as well as the way in which the model's component objects are located within the mould. In turn, the way in which the mould is divided and the arrangement of component elements depend on the geometrical features of the model being fabricated.

Because of the complex shapes of medical objects, it is important to appropriately select and plan the placement of the gating system, i.e. inflow and bleed lines, to ensure access of the casting material to all spaces in the model.

A casting defect (like a cavity) e.g. near the dental root area may result in an improper connection to adjacent components. This phenomenon may lead to dislocation of components, and even to disassembly of the model.

The hybrid method described in this article enabled us to fabricate a multi-object model of properties different from those obtainable with RP techniques. When applied in experimental studies, the discussed methods may reduce costs of model fabrication [28]. Models built with the use of the proposed approaches featured a reliable reconstruction of both internal and external anatomical structures.

The designed approach, under the name "Method of reconstructing medical models with internal structure and the use of materials of diverse properties," was submitted for patent protection (No. P.398644)

\section{Conclusions}

1. The Polyjet Matrix seems to be a very effective technology in reconstructing complex, multi-object anatomical structures. A drawback of the method is the relatively high cost of fabricating a physical model.

2. The presented hybrid method allows us to recreate multi-object and multi-material structures. The application of the method may reduce costs of fabricating models for experimental studies and offers the possibility of using materials of desired properties.

3. The methodology of fabricating multi-object models by combining RP and vacuum casting (VC) techniques was successfully verified using an experimental model of the mandible.
4. The reconstruction of complex multi-object models on the basis of CT data requires the appropriate set-up of acquisition, segmentation and image data processing parameters in order to eliminate artefacts which affect the quality of the generated multi-object model.

5. Materials available in the PolyJet Matrix method impose a restriction on the application of the method in transmitted-light photoelasticity tests. Materials used in the PolyJet Matrix technique can be used in reflected-light photoelasticity tests.

\section{REFERENCES}

[1] P. D. Parchi, V. Ferrari, N. Piolanti, L. Andreani, S. Condino, G. Evangelisti and M. Lisanti, "Computed Tomography Prototyping and Virtual Procedure Simulation in Difficult Cases of Hip Replacement Surgery”, Surg Technol Int. 23, 228-234 (2013).

[2] D. White, K. L. Chelule and B. B. Seedhom, "Accuracy of MRI vs CT imaging with particular reference to patient specific templates for total knee replacement surgery", Int J Med Robot 4 (3), 224-231 (2012).

[33] B. Sanghera, S. Naique, Y. Papaharilaou and A. Amis, "Preliminary study of rapid prototype medical models", Rapid Prototyping Journal 7 (5), 275-284 (2001).

[4] M. Salmi, J. Tuomi, K. S. Paloheimo, R. Björkstrand, M. Paloheimo, J. Salo, R. Kontio, K. Mesimäki and A. Mäkitie, "Patient-specific reconstruction with 3D modeling and DMLS additive manufacturing", Rapid Prototyping Journal 18 (3), 209-214 (2012).

[5] M. E. Hoque, "Advanced applications of rapid prototyping technology in modern engineering", www.intechopen.com (2015).

[6] C. Faur, N. Crainic, C. Sticlaru and C. Oancea, "Rapid prototyping technique in the preoperative planning for total hip arthroplasty with custom femoral components", Wien Klin Wochenschr. 125 (5-6), 144-149 (2013).

[7] A. Mäkitie, K.S. Paloheimo, R. Björkstrand, M. Salmi, R. Kontio, J. Salo, Y. Yan, M. Paloheimo and J. Tuomi, "Medical applications of rapid prototyping three-dimensional bodies for planning and implementation of treatment and for tissue replacement", Duodecim 126 (2), 143-151 (2010).

[8] S. Miechowicz, A. Ciszewski, M. Janiszewski, J. Jamka, M. Libura and P. Konieczny, "Methods of rapid prototyping in preoperative planning in musculoskeletal reconstructive surgery", Chir. Narządów Ruchu Ortop. 76 (2), 63-68 (2011).

[9] M. Salmi, J. Tuomi, R. Sirkkanen, T. Ingman and A. Mäkitie, "Rapid Tooling Method for Soft Customized Removable Oral Appliances", Open Dent Journal 6, 85-89 (2012).

[10] M. Fantini, F. De Crescenzio, L. Ciocca and F. Persiani, "Additive manufacturing to assist prosthetically guided bone regeneration of atrophic maxillary arches", Rapid Prototyping Journal 21 (6), 705-715 (2015).

[11] M. Pawlikowski, K. Skalski and M. Haraburda, "Process of hip joint prosthesis design including bone remodeling phenomenon", Computers and Structures 81 Nos 8/11, 887-893 (2003).

[12] A. Mróz, K. Skalski and W. Walczyk, "New lumbar disc endoprosthesis applied to the patient's anatomic features", Acta of Bioengineering and Biomechanics 17 (2), 25-34 (2013).

[13] J. Domanski, K. Skalski, R. Grygoruk and A. Mróz, "Rapid prototyping in the intervertebral implant design process", Rapid Prototyping Journal, 21 (6), 735-746 (2015). 
[14] A. Mäkitie, J. Korpela, L. Elomaa, M. Reivonen, A. Kokkari, M. Malin, H. Korhonen, X. Wang, J. Salo, E. Sihvo, M. Salmi, J. Partanen, K. S. Paloheimo, J. Tuomi, T. Närhi and J. Seppälä, "Novel additive manufactured scaffolds for tissue engineered trachea research", Acta Oto-Laryngologica 133 (4), 412-417 (2013).

[15] J. Słowiński, "Procedure of generating the individually matched bone scaffolds", Acta of Bioengineering and Biomechanics, 13 (3), 15-21 (2011).

[16] J.D. Curtis, S.D. Hanna, E.A. Patterson and M. Taroni, "On the Use of Stereolitography for the Manufacture of Photoelastic Models", Society for Experimental Mechanics, 43 (2), 23-28 (2003).

[17] D. E. Karalekas and A. Agelopoulos, "On the use of stereolithography built photoelastic models for stress analysis investigations", Materials \& Design, 27 (2), 100-106 (2006).

[18] L. Fan, Q.F. Huang, F.Q. Zhang and Y.P. Xia, "A new method of fabricating photoelastic model by rapid prototyping", Shanghai Kou Qiang Yi Xue. 20 (5), 470-473, (2011).

[19] T. Wohlers, (ed.)., "Wohlers Report 2014 - 3D Printing and Additive Manufacturing State of the Industry. Annual Worldwide Progress Report", http://wohlersassociates.com. (2014).

[20] E. Huotilainen, M. Paloheimo, M. Salmi, K.S. Paloheimo, R. Björkstrand, J. Tuomi, A. Markkola and A. Mäkitie, "Imaging requirements for medical applications of additive manufacturing", Acta Radiologica 55 (1), 78-85 (2014).
[21] T. Kudasik, S. Miechowicz, R. Chrzan and A. Urbanik, "3D reconstruction of complex surfaces of syndesmoses cranii based on the spiral CT and CBCT data", Springer-Verlag, International Journal of Computer Assisted Radiology and Surgery, 6, 265-266, (2011).

[22] http://www.ablesw.com/3d-doctor/(2015).

[23] http://www.stratasys.com (2015).

[24] H. Kopecki, Problemy analizy stanów naprężenia ustrojów $w$ świetle badań eksperymentalnych metodami mechaniki modelowej, Zeszyty Naukowe Politechniki Rzeszowskiej, nr 78, Mechanika, z. 26, Rzeszów, 1992.

[25] M. Kopkowicz, Metody doświadczalne badań konstrukcji, Oficyna Wyd. Politechniki Rzeszowskiej, Rzeszów, 2003.

[26] S. Miechowicz, T. Markowski, T. Kudasik and O. Markowska, "Application of Rapid Protyping Resins for Photoelastic Testing”, DEMI 2011 - International Coference on accomplishments in Electrical and Mechanical Engineering and Information 2011, 247-252 (2011).

[27] M. Salmi, K.S. Paloheimo, J. Tuomi, J. Wolff and A. Mäkitie, "Accuracy of medical models made by additive manufacturing (rapid manufacturing)", Journal of Cranio-Maxillofacial Surgery 41 (7), 603-609 (2013).

[28] E.Atzeni, L. Iuliano, P. Minetola and M. Salmi, "Redesign and cost estimation of rapid manufactured plastic parts", Rapid Prototyping Journal 16 (5), 308-317 (2010). 